\title{
Libros didácticos en la formación de profesores de ciencias: comprendiendo las relaciones entre los saberes académicos y los saberes escolares
}

\author{
Déborah Vidal Vasconcellos* \\ Maria Margarida Gomes** \\ Marcia Serra Ferreira ${ }^{* * *}$ \\ Sandra Escovedo Selles ${ }^{* * *}$
}

Artículo recibido: 6-12-2005 y aprobado:26-04-2006

Resumen: En este trabajo fueron analizados diversos libros didácticos de ciencias para la educación fundamental del profesorado en ciencias y biología, buscando ayudar a los futuros profesores a ampliar su conocimiento sobre la educación escolar. Históricamente, los libros didácticos vienen desempeñando un importante papel en la selección y organización tanto de los contenidos como de los métodos de enseñanza. Diversas críticas vienen siendo dirigidas a estos materiales, sin considerar las características específicas que los distinguen de los libros de texto académicos. Partiendo de esta perspectiva, fueron comparados seis libros didácticos brasileños de ciencias con libros universitarios académicos de bioquímica y de ecología, en los que se tomó como foco del análisis el contenido fotosíntesis. Los futuros profesores en formación analizaron cómo se introducía en los libros el concepto de la fotosíntesis, y su relación con los otros contenidos para identificar diferencias entre los dos conjuntos de libros. Se estableció que los libros didácticos se distinguen de los libros universitarios académicos tanto en lo que se refiere a la selección de los contenidos, como a las ilustraciones empleadas; además, éstos también se diferencian desde el punto de vista de sus propuestas de enseñanza, y el lenguaje empleado en cada uno de los niveles de enseñanza es una forma de identificar estas diferencias. Con la suposición de que los estudiantes universitarios dominan un conjunto de conceptos básicos para comprender la fotosíntesis, los libros académicos emplean un estilo de lenguaje formal. Los dos conjuntos de libros contrastan no sólo en los objetivos educativos, sino también en el abordaje pedagógico, indicando que atienden a finalidades sociales distintas. El análisis efectuado por los estudiantes propició reflexiones acerca de su formación como futuros profesores de ciencias, considerando las diferencias entre el conocimiento académico y el escolar.

Palabras clave: Formación inicial de profesores de ciencias, libros didácticos, fotosíntesis.

\begin{abstract}
Textbooks in the initial science teacher education: understanding the relationships between academic knowledge and school knowledge
\end{abstract}

Abstract: The analysis of school textbooks has been used in this work on an initial teacher education undergraduate course to help future science teachers to deepen their knowledge about education in schools. Historically, school textbooks have had an important role in selecting and organizing not only the content of the curriculum but also the teaching methods. These curriculum materials have been critiqued for their academic characteristics without considering their specific school characteristics. Taking this broader perspective, six Brazilian school textbooks have been compared to two academic textbooks focusing on photosynthesis. The trainee teachers have analyzed the books taking into consideration how the concept of photosynthesis is introduced and related to the other content in order to identify differences between the two sets of textbooks. School textbooks are seen to differ from university ones in the selection of content and illustrations. They also differ in their learning purposes and use informal language according to the level. University students require a common set of basic concepts to understand photosynthesis and the academic textbooks present a formal language style. The two set of textbooks show a contrast not only on the proposed educational aims but also on the approach taken, showing that they are related to different social goals. The analysis undertaken by the trainee teachers allowed them to reflect on how to be prepared for their future work as science teachers by considering the differences between school and academic content knowledge.

Key words: Initial science teacher education, textbooks, photosynthesis.

\footnotetext{
* Instituto de Ciências Biomédicas, Universidade Federal do Rio de Janeiro (UFRJ).deborahvidal@uol.com.br

**Colégio de Aplicação,UFRJ.margaridagomes@mls.com.br

*** Faculdade de Educação, Universidade Federal do Rio de Janeiro (UFRJ). mserra@ufrj.br

${ }^{* * * *}$ Faculdade de Educação, Universidade Federal Fluminense (UFF). escovedoselles@gmail.com
} 


\section{Introducción}

Diversos estudios señalan la necesidad de brindar experiencias en la formación docente inicial, que posibiliten a los futuros profesores profundizar el conocimiento de las características propias del contexto escolar (Ferreira, Vilela y Selles, 2003; Monteiro, 2001). Considerando esta necesidad, el presente trabajo buscó problematizar los libros didácticos ${ }^{1}$ escolares en los procesos de formación profesional. Dichos materiales históricamente han desempeñado un importante papel en la selección y organización de los contenidos y de los métodos de enseñanza (Selles y Ferreira, 2004). Frecuentemente, han sido blanco de numerosas críticas, dejando de ser percibidos en sus especificidades escolares y en su carácter productivo. Si, por un lado, los libros didácticos poseen problemas en el tratamiento de los diversos contenidos escolares, por el otro, argumentamos que los libros de texto universitarios no se constituyen en una alternativa didáctica para el público escolar.

Desde esta perspectiva, el trabajo analiza el modo en que la fotosíntesis es abordada en seis libros didácticos destinados al quinto año de la enseñanza fundamental en Brasil, buscando comprender las especificidades de ese conocimiento escolar, frente a cómo el tema es presentado en dos libros de textos destinados a la enseñanza superior, uno de ecología y otro de bioquímica. Nuestro análisis, sin embargo, difiere de

1 En este artículo, el término "libro didáctico" será empleado para los libros utilizados en las escuelas, mientras que el término "libros de texto" designa a los libros utilizados en las clases universitarias. los estudios ya existentes sobre los libros didácticos, en los que el foco recae predominantemente sobre los errores conceptuales, lo que fue señalado en un trabajo anterior (Ferreira y Selles, 2004). Del mismo modo, se aparta de los estudios que realizan análisis retóricos (Pinto y Martins, 2001; Nascimento, 2003) y de lenguaje (Cassab y Martins, 2001). Nuestra intención fue focalizar el conocimiento expresado en los libros didácticos como constituyente de una cultura escolar (Forquin, 1993) y, por tanto, con características propias y distintas de los saberes académicos y científicos. Desde esta perspectiva, pasamos a valorizar los libros didácticos por sus características y objetivos propios, dejando de lado las fuentes científicas y académicas como las únicas referencias.

Defendemos que una comparación más sistemática entre los materiales destinados a la escuela y a la formación universitaria nos provee de nuevos elementos para reflexionar sobre las finalidades de determinados contenidos -tales como la fotosíntesis- en los currículos de educación básica. De esta manera, dicha comparación, al ser parte de la formación inicial de los profesores, puede ser significativa para la comprensión de los procesos de "didactización" de los conocimientos científicos en los contextos escolares. En las próximas secciones presentamos, en primer lugar, un análisis comparativo del tema de la fotosíntesis en libros didácticos y en libros de texto. A continuación, relacionamos este análisis con los procesos de formación docente inicial.

Análisis de la fotosíntesis en libros 


\section{didácticos y libros de textos}

Nuestro punto de partida fueron las actividades de práctica de la enseñanza, realizadas en 2001, en la carrera de formación inicial de profesores de ciencias y biología, de la Universidad Federal de Río de Janeiro. En estas actividades, los futuros profesores plantearon ideas de los alumnos de educación básica sobre la fotosíntesis (Vasconcellos, Gomes y Ferreira, 2001; 2002a) y, a partir de allí, fueron vivenciados los procesos de selección, organización y traducción de los contenidos y métodos de enseñanza para la temática (Vasconcellos, Gomes y Ferreira, 2002b). El desarrollo de dichas actividades suscitó diversas reflexiones entre los estudiantes acerca de las especificidades del tratamiento de la fotosíntesis en los libros didácticos. Teniendo en cuenta que estos profesores en formación poseen como referencia básica los conocimientos aprendidos en la esfera académica, y que pasan a confrontarlos con las especificidades de la enseñanza de las ciencias para estudiantes del nivel básico, se desarrolló un trabajo de análisis de los contenidos de la fotosíntesis en los libros didácticos y libros de texto, que pudiese explicitar tales diferencias.

La elección de los seis libros didácticos -Alvarenga, Pedersoli, d'Assunção Filho y Gomes, 1999; Bertoldi y Vasconcellos, 2000; Canto, 1999; César, Sezar y Bedaque, 1998; Louzada, Bortolucci y Moreno, 1999; Silva y Fontinha, s/f- tuvo como referencia el hecho de que tales materiales han sido bastante utilizados en las escuelas brasileñas. Por otra parte, la selección de los libros de textos de Voet y Voet (1995) y de Ricklefs (1993) tuvo en consideración el hecho de que, en la Universidad Federal de Rio de Janeiro, la fotosíntesis tiene gran relevancia, en las disciplinas de bioquímica y ecología básica. El análisis realizado por los estudiantes consideró aspectos como el concepto de fotosíntesis, su inserción en la organización de los contenidos, así como su relación con otros temas abordados en estos libros.

En los libros didácticos destinados al quinto año ${ }^{2}$, la fotosíntesis no ocupa necesariamente un lugar destacado; en las universidades, sin embargo, el tema ha sido abordado con materiales de enseñanza destinados a las diversas disciplinas académicas de la carrera de ciencias biológicas. Tal aspecto se evidencia tanto en el espacio ocupado por el tema como por su localización en varios materiales. Los libros didácticos y de textos difieren en la selección de los contenidos, en el lenguaje utilizado y en las formas de ilustración, además del enfoque y de los objetivos propuestos. En los primeros, existe una explícita preocupación por la edad y por el público al que son destinados; en los segundos, el tema es presentado de modo informativo, sin que los autores tengan en cuenta los procesos de construcción de conocimiento de sus supuestos lectores.

En los libros didácticos, la fotosíntesis se encuentra valorizada e introducida de distintas maneras. Solamente en Canto (1999) existe un capítulo dedicado al tema; en los demás, la fotosíntesis se encuentra en uno o más capítulos y relacionada con contenidos de enseñanza diversos. Por ejemplo, en César, Sezar

\footnotetext{
2 En el quinto año, la media de edad de los alumnos brasileños es de aproximadamiente 11 años.
} 
y Bedaque (1998) y en Louzada, Bertolucci y Moreno (1999), la fotosíntesis se encuentra relacionada con el aire; en Bertoldi y Vasconcellos (2000), Canto (1999), Louzada, Bertolucci y Moreno (1999), y en Silva y Fontinha (s/f), aparece relacionada con la cadena alimenticia. Ya en César, Sezar y Bedaque (1998) y en Silva y Fontinha (s/f), la fotosíntesis se asocia con contenidos de los ciclos biogeoquímicos. Finalmente, en Alvarenga, Pedersoli, D’Assução Filho y Gomes (1999), Silva y Fontinha (s/f), con la energía. En estos materiales para la enseñanza, la preocupación con el público destinatario se explicita en las diversas tentativas de aproximación de la fotosíntesis a fenómenos ya conocidos por los alumnos. Así, el tema aparece relacionado con las nociones de alimento y cadena alimenticia, con los componentes del ambiente y con las diferencias entre animales y vegetales.

En el libro académico universitario de bioquímica (Voet y Voet, 1995), la fotosíntesis está incluida en un capítulo que trata sobre el metabolismo de los seres vivos. El libro de ecología (Ricklefs, 1993) no presenta un capítulo específico sobre el tema, aun cuando aparece en la parte dedicada al flujo de energía en los sistemas biológicos, y también en la parte relacionada con el concepto de adaptación. Estas publicaciones parten del principio de que su público destinatario ya posee la capacidad de comprensión de las diversas temáticas en forma autónoma. En Voet y Voet (1995: 626), por ejemplo, es destinado poco espacio al concepto de fotosíntesis, que aparece así definido:

Plantas y cianobacterias secuestran químicamente la energía luminosa por medio de la fotosíntesis, un proceso dirigido por la luz en el cual el gas carbónico es "fijado" para producir carbohidratos $\left(\mathrm{CH}_{2} \mathrm{O}\right)$.

Del mismo modo, la historia de la construcción de este concepto no se extiende más de media página; el foco está en un microabordaje del proceso, que tiene inicio en la estructura de los cloroplastos, pasa por el nivel molecular y llega a una explicación de lo que ocurre con la energía de los electrones. En este momento, el concepto de fotosíntesis es resignificado como:

Un proceso en el cual electrones de moléculas excitadas de clorofila son pasados a través de una serie de aceptores que convierten la energía de estos electrones en energía química (Voet y Voet, 1995: 634).

En el libro académico universitario de ecología, también es escaso el espacio que se le otorga a la historia de la construcción del concepto de fotosíntesis. En forma análoga al libro de bioquímica, la fotosíntesis también es explicada por medio de un microabordaje:

Las plantas capturan la energía luminosa y la transforman en energía química de ligación en los carbohidratos. La glucosa y otros compuestos orgánicos (almidón y aceites, por ejemplo) pueden ser transportados a través de las plantas o almacenados convenientemente para posterior liberación de la energía por la respiración (Ricklefs, 1993: 87).

Sin embargo, vemos que en el libro de ecología ese microabordaje es relacionado con la comprensión de macroaspectos del proceso:

La fotosíntesis remplaza los bloques de construcción de los carbohidratos y la energía que la planta precisa para sintetizar tejidos y crecer. Reacomo- 
dadas y montadas, las moléculas de glucosa se tornan grasa, aceites y celulosa (Ricklefs, 1993: 87).

Este texto de ecología se diferencia del de bioquímica porque establece relaciones con otros aspectos de los sistemas biológicos, teniendo así un carácter más informativo. Como veremos posteriormente, en tal aspecto, el libro de ecología analizado se aproxima mejor a los libros didácticos, ya que trata más ampliamente el concepto de fotosíntesis. Ésta es resignificada de forma más extensa, debido a que se ocupa tanto del flujo de energía en los sistemas biológicos como de la idea de adaptación, conforme a los dos ejemplos que destacamos:

Las plantas capturan la energía luminosa y la transforman en energía química de ligación en los carbohidratos. La glucosa y otros compuestos orgánicos (almidón y aceites, por ejemplo) pueden ser transportados a través de las plantas o almacenadas convenientemente para posterior liberación de su energía por la respiración (Ricklefs, 1993: 87).

Compare las hojas de los árboles de bosques deciduos con aquellos de especies del desierto. Las primeras son típicamente largas y finas, proporcionando una gran área superficial para absorción de luz y también, inevitablemente, para pérdida de agua (Ricklefs, 1993: 75).

El abordaje de la fotosíntesis en los libros didácticos demuestra cierta similitud con la del libro de ecología. En los primeros, la preocupación con la faja etaria y el público destinatario se explicita en las diversas tentativas de aproximación de la fotosíntesis a fenómenos ya conocidos por los alumnos. Así, el tema aparece relacionado con las nociones de alimento y cadena alimenticia, con los componentes del ambiente y con las diferencias entre animales y vegetales, como se puede observar en los ejemplos que se presentan a continuación:

Tú ya sabes que todo ser vivo necesita energía para sobrevivir. Los animales consiguen esa energía de los alimentos que ingieren. Pero, ¿qué sucede con las plantas? (Canto, 1999: 17).

Y los animales, ¿de dónde consiguen los materiales para crecer? Como no hacen fotosíntesis, se alimentan de los seres clorofilados (animales herbívoros) o de otros animales (animales carnívoros) (Silva y Fontinha, s/f: 31).

Estas relaciones son trabajadas de forma aún más enfática en las definiciones de fotosíntesis que los libros didácticos presentan, evidenciando las diferencias en los objetivos vinculados a la enseñanza de esta temática en los dos niveles:

Los científicos descubrieron que el alimento de las plantas es un tipo de azúcar fabricado por la propia planta. Para producir ese azúcar, hay tres factores de los que el vegetal necesita: agua, luz y un gas existente en el aire llamado gas carbónico. El proceso de fabricación de azúcar por la planta es denominado fotosíntesis. Ella ocurre principalmente en las hojas de los vegetales (Canto, 1999: 18).

Por medio de la fotosíntesis, los seres clorofilados producen azúcares consumiendo gas carbónico y agua del ambiente. En otras reacciones químicas, en las que también pueden necesitar sales minerales, producen una enorme variedad de sustancias 
orgánicas como grasas, proteínas, vitaminas y ácidos nucleicos. Como son los únicos capaces de hacer esto, los seres clorofilados son conocidos como seres productores de alimentos (Silva y Fontinha, s/f: 30).

Todas las cadenas alimenticias comienzan con el ser vivo que capta la energía solar. Esa energía es usada por las plantas, algas y algunas bacterias en el proceso de la fotosíntesis. Por ser capaces de producir su propio alimento, decimos que esos seres son los productores en un ecosistema.

Para generar su alimento, los productores dependen de la clorofila, pigmento que absorbe la energía de la luz solar. Utilizando esa energía en conjunto con el gas carbónico, el agua y las sales minerales en ella disueltos, los productores fabrican azúcar, proteínas y grasas. Tales sustancias son usadas por las plantas y algas como material para su propio crecimiento y como reserva de energía (Bertoldi y Vasconcellos, 2000: 177).

Al analizar estas definiciones, es posible percibir que los libros didácticos presentan sus contenidos en relación con diversos aspectos de la vida de los estudiantes, tales como: edad, conocimientos previos y comprensión general del mundo natural. Optan por abordar los contenidos de fotosíntesis, enfocando la relación con el mundo cotidiano de los alumnos. También puede ser identificada cuando los autores de estos materiales buscan establecer un diálogo que privilegie la utilidad -y no los aspectos más abstractos- de los asuntos que los alumnos están aprendiendo. En este sentido, estos libros revelan poseer especifidades y características propias de los contextos escolares.
El análisis comparativo entre los libros de texto y los libros escolares indica que, en el abordaje de los conocimientos escolares sobre fotosíntesis, los libros didácticos de quinto año poseen mayores aproximaciones al libro académico de ecología. Tal aspecto puede estar relacionado con el hecho de que el abordaje que hace el de ecología analizado contempla más los aspectos explicativos macro sobre el tema, lo que lo aproxima a las estrategias escolares de enseñanza. Podemos sostener la hipótesis de que tal abordaje ha sido seleccionado por los autores de los libros didácticos de ciencias, porque permite establecer mayores referencias con los aspectos cotidianos de los alumnos y, por esta razón, se ajustan mejor a las finalidades educativas de la escuela.

\section{Los libros didácticos en la formación de profesores: la comprensión de un conocimiento sui géneris}

Al iniciar las disciplinas pedagógicas, los estudiantes traen una comprensión de los conocimientos científicos basada en los estudios de textos académicos que utilizaron a lo largo de la carrera universitaria. De esta forma, sus expectativas en relación con la enseñanza de los conocimientos escolares en ciencias tiene como base la simple reproducción de contenidos académicos. Solamente cuando pasan a cursar las referidas disciplinas pedagógicas, los futuros profesores inician una reflexión acerca de aspectos particulares de la escuela y, más especificamente, de la enseñanza de ciencias y biología.

La formación profesional se extiende más allá de los aprendizajes construidos durante las carreras de formación inicial; 
sin embargo, reconocemos que el período dedicado al aprendizaje práctico de la profesión imprime importantes marcas en la construcción de una identidad docente. Coincidimos con Monteiro (2001) cuando afirma que la preparación para las actividades docentes, en el ámbito de la formación inicial, ocurre en un momento estratégico, una especie de ritual de iniciación, en el que los futuros profesores de ciencias y biología tienen la posibilidad de interactuar con los diferentes sujetos que participan de este proceso. La singularidad de este momento se debe, entre otros factores, al hecho de que los estudiantes pueden vivenciar experiencias docentes de forma tutelada -algo que no se repetirá más, cuando estén en el ejercicio pleno de sus actividades profesionales-, contando con interlocutores que los ayudan a reflexionar acerca de los materiales didácticos, de los modos de enseñanza, de las respuestas de los niños a las clases que dictan y, sobre todo, de los límites institucionales que interfieren en el desarrollo del trabajo docente.

Apoyados por el profesor universitario, e interactuando con los profesores de las escuelas, a medida que los estudiantes pasan a crear sus propias actividades docentes, van dándose cuenta de las características propias del contexto escolar, así como de la necesidad de resignificar los contenidos y los métodos de enseñanza, aprendidos a lo largo de su formación académica. De ahí deriva la importancia de que los futuros profesores tengan oportunidades para problematizar materiales como los libros didácticos, buscando identificar los diversos fundamentos, que están más allá de los conocimientos científicos, y que son importantes en la producción de estos textos.

La formación de profesores de ciencias que defendemos se apoya en la contribución de Tardif y colaboradores (por ejemplo, Tardif, Lessard y Lahaye, 1991; Tardif y Lessard, 2002; Tardif, 2005; Gauthier et al., 1998) que confieren peso tanto al entendimiento de la naturaleza de los saberes que sirven de base para la realización del trabajo docente, como a las fuentes desde las que se producen. Para Tardif (2002:223) no es posible comprender los saberes docentes sin relacionarlos con los condicionantes y con el contexto de trabajo, pensándolos como una construcción social producida por la racionalidad concreta de los actores, por sus deliberaciones, racionalizaciones y motivaciones, que constituyen la fuente de sus juicios, elecciones y decisiones.

Entender la formación inicial desde la perspectiva de los saberes docentes implica replantear las estrategias formativas de los cursos de preparación profesional. De modo más amplio, esta perspectiva nos permite reflexionar sobre la natureza y el alcance del aprendizaje docente, en los límites temporales impuestos por la duración de los programas de formación del profesorado y sobre el conjunto de necesidades formativas, tanto las que se refieren a los dominios del contenido específico como a las que se relacionan con los condicionantes propios de la escuela. Tales condicionantes se expresan por medio del tiempo y del espacio que organizan la institución escolar, y que difieren, de modo significativo, del tiempo y del espacio de la formación inicial docente (Ferreira, Vilela y Selles, 2003). El apren- 
dizaje en estos condicionantes exige una inversión teórica que sitúe la enseñanza de las disciplinas ciencias y biología en el contexto escolar, dejando de percibirlas apenas como una expresión reducida de sus ciencias de referencia. Por ejemplo, al confrontar sus experiencias más académicas sobre el tema de la fotosíntesis con su formato en los contextos escolares, los estudiantes pueden iniciar su propia comprensión de las propiedades específicas de los conocimientos que caracterizan la escuela.

Las importantes contribuciones teóricas provenientes de los estudios sobre la formación docente son enriquecidas por aquellas que explicitan la vinculación entre las instituciones formadoras y el ambiente escolar. Vemos allí algunas posibilidades para fortalecer las relaciones de aprendizaje docente emprendidas por nuestros estudiantes en los ambientes escolares durante el período de la práctica, cuando pasan a analizar los materiales curriculares empleados en la enseñanza de ciencias y biología. Estas perspectivas ofrecen elementos para interpretar y encontrar nexos históricos entre la producción de estos materiales y su uso en las prácticas docentes.

Si por un lado esta perspectiva de la formación docente se apoya en los estudios que focalizan los saberes movilizados por los profesores de ciencias y biología a lo largo de su ejercicio profesional, por otro, se sustenta en contribuciones teóricas que caracterizan los procesos didáctico-sociales que transforman los contenidos científicos en contenidos escolares. Apoyándose en los estudios de Chevallard (1991) dice Forquin (1992:32):
La educación escolar no se limita a hacer una selección entre los saberes y los materiales culturales disponibles en un momento dado en una sociedad. Ella debe también, a fin de tornarlos efectivamente transmisibles, efectivamente asimilables para las jóvenes generaciones, entregarse a un inmenso trabajo de reorganización, de reestruturación, de "transposición didáctica".

Siguiendo a este autor, las responsables de la presente investigación sostienen que es necesario tener claridad acerca de los dispositivos mediadores, identificados también por Forquin como mecanismos de didactización, que transforman los objetos por enseñar en objetos de enseñanza. La necesidad funcional de didactización produce un conjunto de características morfológicas típicas de los saberes escolares, que son identificables en ciertas situaciones de comunicación no escolar, denotando la impregnación del espíritu escolar en la cultura de ciertos individuos o de ciertos grupos.

Forquin (1992: 35) ve este proceso materializándose en la escuela, y entiende esta institución como una verdadera productora de configuraciones cognitivas propias, el elemento nuclear de una cultura escolar sui géneris. Esta configuración específica viene recibiendo la atención de los estudiosos de los saberes escolares, que señalan su diferenciación en relación con los saberes científicos, y ven las disciplinas escolares no meramente como una adaptación para fines de enseñanza (Lopes, 2000:145). En esta perspectiva, pasamos a valorizar los libros didácticos por sus características y objetivos propios, dejando de utilizar 
como únicas referencias las fuentes científicas y académicas.

Comprendemos, por tanto, que este trabajo de análisis de los libros didácticos en la formación inicial docente posibilita reflexiones acerca de las singularidades propias de los conocimientos escolares frente a los conocimientos académicos aprendidos en las carreras universitarias. De esta forma, las actividades que permitan a los futuros profesores confrontar diferencias y proximidades entre las configuraciones de conocimientos, típicas de los libros didácticos y de los libros de texto, pueden facilitarles la comprensión de la dinámica de la práctica docente. Ésta incluye la asunción de un saber construido en la interlocución entre los saberes académicos y los saberes circulantes en las instancias escolares. Podemos citar como ejemplo la comprensión del concepto de fotosíntesis en los libros escolares y de sus relaciones con las finalidades de enseñanza de la disciplina de ciencias.

Es importante considerar y caracterizar las exigencias de didactización, y encontrar sus marcas en los materiales de enseñanza que los profesores emplean. Como afirma Verret (Forquin, 1992:33), en la transformación de los saberes escolares los procesos didácticos se benefician de todas las protecciones que justamente le faltan al investigador:

Protección contra los errores e impasses de las investigaciones mal encaminadas (la transmisión didáctica descarta la transmisión de las investigaciones no exitosas); protección contra las discontinuidades de las investigaciones interrumpidas (la transmisión didáctica presupone la transmisión histórica exitosa de las investigaciones exitosas); protección contra la dispersión en la aprehensión del objeto (la transmisión didáctica estructura la investigación sobre sus momentos fuertes, haciendo con esto economía del detalle) [el destacado es nuestro].

Corroborando esta forma de entender los procesos escolares, Izquierdo, Sanmartí y Mariona (1999:45) buscan aplicarla a la enseñanza de las ciencias:

Si las ciencias son el resultado de una actividad humana compleja, su enseñanza no puede serlo menos: debe concebirse también como actividad, y para ello debe tener la meta, el método y el campo de aplicaciones adecuados al contexto escolar, conectando con los valores del alumnado y con el objetivo de la escuela (que es promover la construcción de conocimientos y hacerlos evolucionar).

En el análisis de los libros didácticos identificamos algunos mecanismos de didactización que se valen de estas protecciones y producen un conocimiento con configuración propia. Así, parece relevante reconocer, en estos libros, algunos rasgos de la actividad científica que permanecen, y aquellos que, reinventados en función de la economía del detalle, constituyen configuraciones distintas del conocimiento proveniente de la actividad científica. Destacamos algunos ejemplos cuando, en los libros didácticos de ciencias investigados, las referencias a las investigaciones bioquímicas son expresadas sin muchos detalles:

Los científicos descubrieron que el alimento de las plantas es un tipo de azúcar fabricado por la propia planta (Canto, 1999: 18). 
Así mismo, cuando etapas y procesos intermediarios de la cadena fotosintética son genéricamente registrados como:

En otras reacciones químicas, en las cuales también pueden necesitar sales minerales, producen una enorme variedad de sustancias orgánicas como grasas, proteínas, vitaminas y ácidos nucleicos (Silva y Fontinha, s/f: 30).

Estos ejemplos, extraídos de los libros didácticos de ciencias, ilustran cómo las finalidades de la escolarización predominan en los procesos sociohistóricos que producen el conocimiento escolar. Comprendemos que esta configuración constituye un caso de "economía del detalle”, conforme cita Forquin. En esta interpretación, exponemos la singularidad del conocimiento escolar al distinguirlo del texto científico, y nos alejamos de las interpretaciones que conciben los ejemplos mencionados como una mera "reducción para fines de enseñanza", una omisión de carácter conceptual o una ausencia de historicidad que expone fragilidades epistemológicas graves.

\section{Conclusiones}

En un estudio anterior (Ferreira y Selles, 2004), destacamos que gran parte de las investigaciones con libros didácticos brasileños se ocupa de problematizarlos, señalando principalmente sus deficiencias en relación con los aspectos conceptuales y filosóficos de las ciencias de referencia, analizando poco los aspectos productivos de estos materiales. Tal perspectiva nos orientó en el análisis desarrollado con los estudiantes y, de esta forma, fue posible superar una visión centrada sólo en la detección de errores conceptuales. En el caso específico de la fotosíntesis, pudimos percibir que los libros didácticos, más que poseer errores, difieren de los libros académicos tanto en el enfoque como en los objetivos propuestos, evidenciando que fueron producidos para instancias culturales propias y atendiendo a finalidades sociales relacionadas con los procesos de escolarización.

Cabe al profesor, por tanto, conociendo estas diferentes instancias -sea cual sea su instancia de formación inicial o aquella en la que ejercerá su oficio-, apropiarse de los saberes académicos y escolares en la producción de un trabajo original y adecuado a su realidad escolar. Defendemos que los procesos formativos docentes que se orientan a partir de tal perspectiva colocan a los estudiantes en la posición de interlocutores entre los saberes movilizados tanto en la universidad como en el contexto escolar. En este sentido, los trabajos con libros didácticos son referencias importantes para este complejo e incitante aprendizaje del hacer docente. $\boldsymbol{\Delta}$

\section{Bibliografía}

Alvarenga, Pedersoli, D’assunção Filho y Gomes (1999). Ciências Integradas. Vol. 1. Belo Horizonte: Dimensão. 
Bertoldi O.B., y Vasconcellos (2000). Ciência y Sociedade. $5^{\mathrm{a}}$ série. São Paulo: Scipione.

Canto, E. L. (1999). Ciências Naturais: Aprendendo com o Cotidiano. $5^{\mathrm{a}}$ série. São Paulo: Moderna.

Cassab, M. y Martins, I. (2001). “A linguagem no livro didático de Ciências: um exercício de análise". En: Anais do I Encontro Regional de Ensino de Biologia. Niterói: FE/UFF e SBEnBio RJ/ES.

César, Sezar y Bedaque (1998). Ciências: Entendendo a Natureza. O Mundo em que Vivemos. São Paulo: Saraiva.

Chevallard, Y. (1991). La transposición didáctica - Del saber sabio al saber enseñado. Buenos Aires: Aique.

Ferreira, M. S., Vilela, M. L. y Selles, S. E. (2003). "Formação docente em Ciências Biológicas: estabelecendo relações entre a Prática de Ensino e o contexto escolar". En: Ferreira, M. S. y Selles, S. E. (orgs.), Formação Docente em Ciências: Memórias e Práticas. Niterói: EDUFF, pp. 29-46.

y Selles, S. E. (2004). “Análise de livros didáticos em ciências: entre as ciências de referência e as finalidades sociais da escolarização". Educação em Foco. Vol. 8 (2), 63-78.

Forquin, J. C. (1992). "Saberes escolares, imperativos didáticos e dinâmicas sociais". Teoria e Educação. nº. 5, pp. 28-49.

(1993). Escola e Cultura: as bases sociais e epistemológicas do conhecimento escolar. Porto Alegre: Artes Médicas.

Gauthier, C., Martineau, S., Desbiens, J. F., Malo, A. y Simard, D. (1998). Por uma teoria da pedagogia: pesquisas contemporâneas sobre o saber docente. Ijuí: Editora Unijuí.
Izquierdo, M, Sanmartí, N. y Mariona, E. (1999). "Fundamentación y diseño de las prácticas escolares de ciencias experimentales. Enseñanza de las Ciencias, 17 (1), 45-59.

Lopes, A. C. (2000). “Organização do Conhecimento Escolar: analisando a disciplinaridade e a integração". En Linguagens, espaços e tempos no ensinar e aprender. Encontro Nacional de Didática e -Prática de Ensino (Endipe), DP\&A, Rio de Janeiro, pp.147-162.

Louzada, F. N., Bortolucci, J. A. y Moreno, C. R. C. (1999). Ciências: Natureza, Tempo e Espaço. $5^{\mathrm{a}}$ série Belo Horizonte: Lê.

Monteiro, A. M. (2001). “A Prática de Ensino e a produção de saberes na escola”. En: Candau, V. (org.), Didática, Currículo e Saberes Escolares. Rio de Janeiro: DP\&A, pp. 129-147.

Nascimento, T. G. (2003). O texto de genética no livro didático de Ciências: uma análise retórica. Dissertação de Mestrado. Rio de Janeiro: NUTES/UFRJ.

Pinto, G. A. y Martins, I. (2001). "Evolução nos livros didáticos de Biologia: uma análise retórica". En: Anais do I Encontro Regional de Ensino de Biologia. Niterói: FE/UFF e SBEnBio RJ/ES.

Ricklefs, R. E. (1993). A Economia da Natureza. $3^{a}$. ed. Editora GuanabaraKoogan .

Selles, S. E. y Ferreira, M. S. (2004). "Influências histórico-culturais nas representações sobre as estações do ano em livros didáticos de Ciências". Ciência \& Educação 10 (1), 101, 110. Bauru: UNESP. 
Silva, P. M. y Fontinha, S. R. (s/f). Vol. 1. A Terra, um Planeta Vivo. São Paulo: Nacional.

Tardif, M. (2002). Saberes docentes e formação profissional. Petrópolis: Vozes.

y Lessard, C. (2005). O trabalho docente. Elementos para uma teoria da docência como profissão de interações humanas. Petrópolis: Vozes. . y Lahaye, L. (1991). "Esboço de uma problemática do saber docente”. Teoria \& Educação. Vol. 1, nº. 4, pp. 215-233.

Vasconcellos, D. V., Gomes, M. M. y Ferreira, M. S. (2001). "Idéias de alunos da quinta série sobre nutrição vegetal: subsídios para o trabalho docente". En: Anais do I Encontro Regional de Ensino de Biologia. Niterói: FE/UFF e SBEnBio RJ/ES. (2002a). "Fotossíntese e respiração: as idéias dos alunos de quinta série como ponto de partida para o planejamento de ensino". En: Coletânea do VIII Encontro Perspectivas do Ensino de Biologia. São Paulo: FE/USP.

"Planejamento e regência de atividades de ensino na formação docente: o exemplo da fotossíntese na quinta série do CAp/UFRJ". En: Coletânea do VIII Encontro Perspectivas do Ensino de Biologia. São Paulo: FE/USP.

Voet, D. y Voet, J. G. (1995). Biochemistry. $2^{a}$. ed. Nueva York: John Wiley \& Sons, Inc. 